\title{
Alu pair exclusions in the human genome
}

\author{
George W Cook', Miriam K Konkel', James D Major III', Jerilyn A Walker', Kyudong Han² and Mark A Batzer ${ }^{1 *}$
}

\begin{abstract}
Background: The human genome contains approximately one million Alu elements which comprise more than $10 \%$ of human DNA by mass. Alu elements possess direction, and are distributed almost equally in positive and negative strand orientations throughout the genome. Previously, it has been shown that closely spaced Alu pairs in opposing orientation (inverted pairs) are found less frequently than Alu pairs having the same orientation (direct pairs). However, this imbalance has only been investigated for Alu pairs separated by 650 or fewer base pairs (bp) in a study conducted prior to the completion of the draft human genome sequence.

Results: We performed a comprehensive analysis of all $(>800,000)$ full-length Alu elements in the human genome. This large sample size permits detection of small differences in the ratio between inverted and direct Alu pairs (I:D). We have discovered a significant depression in the full-length Alu pair I:D ratio that extends to repeat pairs separated by $\leq 350,000 \mathrm{bp}$. Within this imbalance bubble (those Alu pairs separated by $\leq 350,000 \mathrm{bp}$ ), direct pairs outnumber inverted pairs. Using PCR, we experimentally verified several examples of inverted Alu pair exclusions that were caused by deletions.

Conclusions: Over 50 million full-length Alu pairs reside within the I:D imbalance bubble. Their collective impact may represent one source of Alu element-related human genomic instability that has not been previously characterized.
\end{abstract}

\section{Background}

Retrotransposons are mobile DNA elements that populate genomes via their respective RNA transcripts. The retrotransposon with the highest copy number in the human genome is the Alu element [1]. Alu elements lack the necessary repertoire of enzymes to effect their independent insertion and are thus classified as nonautonomous mobile elements. For recent reviews, see [2-4].

Following transcription, Alu RNA is thought to require the assistance of the LINE1 open reading frame 2 protein (ORF2p) both for nicking the genome at the insertion site and for reverse transcription of the $A l u$ RNA transcript $[5,6]$. The endonuclease and reverse transcriptase functions of ORF2p are referred to as L1EN and L1RT, respectively. While L1EN has been shown to have some tolerance for target site variation, it most frequently cleaves at the T/A transition within the sequence, 5'-TTTTAA-3' [7-10]. Following cleavage, the

\footnotetext{
* Correspondence: mbatzer@|su.edu

'Department of Biological Sciences, Louisiana State University, Baton Rouge, Louisiana, 70803, USA

Full list of author information is available at the end of the article
}

poly- $\mathrm{T}$ sequence of the target site becomes accessible to the complementary poly(A) tail of Alu RNA. Hybridization of these two sequences results in a short RNADNA hybrid that both orients the RNA transcript and primes reverse transcription of the Alu RNA by L1RT. Identical sequences flanking the insertion are characteristic of most Alu elements [11]. These flanking sequences are referred to as target site duplications (TSDs) $[2,12]$. The presence of TSDs suggests that a nick occurs on the complementary strand of DNA 3' to the L1EN cleavage site on the first strand. However, litthe is known of the mechanisms associated with this second nick or the eventual insertion of the 5' end of the Alu element $[13,14]$. This process of Alu element mobilization and insertion is commonly referred to as target primed reverse transcription (TPRT) $[15,16]$. TPRT also occurs with two additional non-long terminal repeat (LTR) retrotransposons, LINE1 and SVA (SINE-R, variable number of tandem repeats and $A l u$ ) elements, within the human lineage [8]. While recognizing rare exceptions $[17,18]$, the majority of non-LTR retrotransposon insertions are dependent upon the activity of L1EN. As with Alu elements, LINE1 and SVA element
C Biomed Central

() 2011 Cook et al; licensee BioMed Central Ltd. This is an Open Access article distributed under the terms of the Creative Commons Attribution License (http://creativecommons.org/licenses/by/2.0), which permits unrestricted use, distribution, and reproduction in any medium, provided the original work is properly cited. 
insertions are typically characterized by TSDs that flank each element.

Alu elements also possess several features that provide directionality. Including the poly(A) tail, full-length $A l u$ elements are approximately $300 \mathrm{bp}$ in length (Additional File 1, Figure S1) and are dimeric structures with two adenine-rich regions flanking the 3' monomer [2,19]. The middle adenine-rich region separates the two monomers and the 3 ' adenine-rich region forms the variable length poly(A) tail. Additionally, the 5' monomer possesses the $\mathrm{A}$ and $\mathrm{B}$ boxes required for the transcription by RNA Polymerase III and the 3' monomer contains a 31-bp insert not present in the 5 ' monomer $[20,21]$.

Inverted pairs of full-length Alu elements form nearpalindromic sequences that are separated by spacers of other DNA sequences of varying size and composition. Palindromic sequences have been shown to be unstable in Escherichia coli [22], yeast [23] and mice [24]. The genomic instability of inverted Alu pairs has also been demonstrated in a yeast experimental system [25]. Other previous research has reported that inverted Alu pairs are potential sources of chromosomal instability when separated by $\leq 650 \mathrm{bp}$ in humans [26]. The ability of Alu sequences to interact is directly correlated with the degree of sequence identity between the copies [25]. It is estimated that the majority of full-length human $\mathrm{Alu}$ elements share sequence identity ranging between 65 and 85 percent [26].

Alu element insertions have been linked to several genetic diseases including hemophilia, hypercholesterolemia and various cancers $[4,27]$. While multiple diseases have been attributed to Alu element insertions, their most important role may be in shaping human genome architecture through various post-insertion interactions. Such interactions could result in deletions, duplications, inversions and a host of other complex genomic structural changes $[9,28]$. Alu element interactions with each other have been found to generate recombination mediated deletions and inversions $[10,29,30]$. In addition, Alu elements have been associated with multiple deletions related to various cancers $[8,31]$ and copy number variation breakpoints [9,32-35].

It has also been shown in humans that closely spaced adjacent $A l u$ pairs in opposing orientation (inverted pairs) are found less frequently than Alu pairs having the same orientation (direct pairs) [26]. However, this imbalance has previously only been investigated for $\mathrm{Alu}$ pairs separated by $\leq 650 \mathrm{bp}$ in a study conducted prior to the completion of the draft human genome sequence. Here, we have performed a comprehensive analysis of all (> 800,000) full-length Alu elements (275 to $325 \mathrm{bp)}$ in the public human genome assembly (hg18). Using the large data set of full-length $A l u$ elements enabled us to detect small imbalances in the ratio between inverted and direct Alu pairs (I:D). We report a potential new insight into human genomic instability, a non-random depression in the I:D ratio for full-length $A l u$ pairs whose elements are separated by up to $350,000 \mathrm{bp}(P<$ 0.05). Over 50 million $(59,357,435)$ full-length Alu pairs reside within this I:D imbalance window. This phenomenon of full-length Alu pair I:D imbalance is hypothesized to reflect the activity of four separate mechanisms which result in $A l u$ pair exclusions (APEs).

\section{Results}

The size distribution of the human genomic Alu element population is shown in Additional File 1, Figure S1. Full-length Alu elements, having lengths between 275 and $325 \mathrm{bp}$, account for approximately 69 percent of all human Alu elements. Slightly over two percent of human Alu elements have lengths greater than $325 \mathrm{bp}$ with 29 percent being truncated ( $<275 \mathrm{bp})$. Sequences of less than 30 bp cannot be reliably determined to be actual $A l u$ elements and are therefore excluded from this study $(P<0.05)$. Alu element length constraints provide a full-length Alu element sample size of 806,880 (Methods).

The directionality of Alu elements creates four possible types of Alu pairs (Additional File 1, Figure S2). Two of these four configurations share both elements in the same (or direct) orientation and two share elements in the opposite (or inverted) orientation. A pair of Alu elements in which both members of the pair are positioned on the positive strand are in the 'forward' orientation. Conversely, when both members in the pair are positioned on the negative strand, the pair is defined as being in the 'reverse' orientation. Throughout this manuscript, the sequence separating each pair is referred to as the spacer. When an inverted Alu pair is oriented with the poly(A) tails pointing toward each other, the pair is termed as being in the 'tail-to-tail' orientation, and when an inverted pair is oriented with the poly(A) tails pointing away from each other, it is termed as being in the 'head-to-head' orientation.

\section{I:D ratio for adjacent full-length Alu pairs departs from unity}

Departures from unity in the full-length $A l u$ pair (FAP) I:D ratio may be suggestive of non-random insertion or deletion of Alu elements within the human genome. Testing for randomness was performed using binomial distributions assuming an equal probability for $\mathrm{Alu}$ insertions to occur on both the positive and negative strands (Methods). Adjacent FAPs contain no Alu elements within the spacer. The human adjacent FAP population of 560,485 contains 252,748 inverted pairs and 307,737 direct pairs. The I:D ratio for this 
population is 0.8213 . Any I:D ratio outside of 0.9947 to 1.0053 reflects a non-random distribution $(P<0.05)$. The I:D ratio for adjacent FAPs of 0.8213 represents a $P$-value of $<0.000001$ and therefore falls well outside of the 95 percent confidence interval for randomness.

Furthermore, the adjacent FAP I:D ratio departure from unity appears to be a function of the FAP spacer size. The median spacer size for adjacent FAPs is 930 bp (mean spacer length $=921 \mathrm{bp}$ ). Adjacent FAPs with less than and greater than this median spacer length possess I:D ratios of 0.7105 and 0.9477 , respectively. The expected I:D range for a random distribution of these half-size FAP populations is 0.9925 to $1.0075(P<$ $0.05)$. A more thorough analysis of the variation of FAP I:D ratio versus spacer size requires adjustment of the data set and is provided later in this section (see CLIQUES, catenated L1EN induced queues of uninterrupted Alu, LINE1 and SVA elements).

The adjacent FAP I:D imbalance calculation reported above provides a macroscopic view of the entire human genome. Human chromosome one was chosen to determine if a similar I:D bias (non-random distributions of Alu elements with respect to orientation) was evident across a smaller region of the genome. A comparison of the actual distribution versus a simulated random distribution of $A l u$ elements on chromosome one indicated that orientational clustering of $A l u$ elements occurs over 40 percent more frequently than would be expected if $A l u$ insertions were orientationally random (Additional File 1, Figure S3).

\section{Three patterns of I:D ratio}

Figure 1A illustrates the I:D ratio for adjacent human FAPs which are separated by $\leq 500 \mathrm{bp}$. This range includes over one-third of the human adjacent FAP population and is the first breakdown of this I:D parameter by individual spacer length. Three distinct patterns of FAP density and I:D ratio are evident from Figure 1.

The first pattern is the combined high FAP density and low I:D ratio (0.073) for spacer lengths of $\leq 24 \mathrm{bp}$. An unexpected inflection point in the frequency of direct FAPs occurs after as spacer size of 6 bp (Figure 1A). This pattern may be indicative of a potential orientational insertion preference for $A l u$ elements within the TSD of an existing $A l u$ element. The second FAP I:D ratio pattern evident in Figure 1A (magnified in Figure 1B) is the 13 bp span of elevated FAPs in the head-to-head orientation within the spacer size range of 24 to $36 \mathrm{bp}$. This span contains 1.6 percent of adjacent human FAPs and is the only spacer size range within the human genome where the FAP I:D ratio exceeds unity (I:D = 1.053). Previous research identified an elevated presence of $A l u$ pairs (> $275 \mathrm{bp}$ ) in this orientation for the spacer size range of 21 to $40 \mathrm{bp}$ [26]. As can be seen in Figure 1B, the most accentuated head-to-head frequencies occur between spacer lengths of 24 to $36 \mathrm{bp}$. For this span of spacer sizes, head-to-head (inverted) FAPs outnumber either forward or reverse (direct) FAPs. Although the most elevated head-to-head frequencies reside within the spacer size range of 24 to $36 \mathrm{bp}$, Figure $1 \mathrm{~B}$ also reveals that an attenuated elevation of head-to-head FAPs over tail-to-tail inverted FAPs is present within the spacer size range of 37 to $50 \mathrm{bp}$.

The third FAP density and I:D ratio pattern is evident in Figure 1A. It is characterized by similar FAP frequencies among the four $A l u$ pair types between spacer sizes of 51 to $500 \mathrm{bp}$. This third pattern persists for adjacent FAPs with spacer sizes of $>500$ bp (data not shown).

\section{CLIQUEs, catenated L1EN induced queues of uninterrupted Alu, LINE1 and SVA elements}

The common dependence of Alu, L1NE1, and SVA insertions upon L1 enzymes raises the possibility that the clustering of closely spaced Alu elements ( $\leq 50 \mathrm{bp}$ ) observed in Figure $1 \mathrm{~A}$ is also associated with various combinations of all three element types. A similar clustering pattern exists in the form of catenated Alu clusters (see Additional File 1, Catenated Alu Clusters and Figure S4). A total of 412,380 various combinations of these $A l u$-LINE1-SVA clusters are present within the human genome. These clusters comprise 16.6 percent of all human DNA and contain 52.6 percent of the Alu, LINE1 and SVA sequence within the human genome. Retrotransposons residing within these L1EN-induced clusters can exist in both orientations but exhibit a clear bias for one orientation. These clusters are characterized by this orientational bias as the I:D ratio for adjacent FAPs within these clusters is 0.3847 . These clusters are enriched with potential L1EN target sites because of their shared TPRT insertion mechanism creating L1ENinduced TSDs flanking these three types of retrotransposons, as well as by the adenine-rich region within Alu elements (see Discussion, APE mechanisms). This enrichment of potential L1EN target sites inherently increases the likelihood of future Alu, LINE1 and SVA elements within these clusters. The common participation of Alu, L1NE1, and SVA elements within catenated clusters is consistent with L1EN activity. These catenated L1EN induced queues of uninterrupted $A l u$, L1NE1, and SVA elements are hereafter referred to as CLIQUEs.

The potential for TPRT-related insertion bias within TSDs makes CLIQUE identification an important consideration in evaluating deviations from unity in the FAP I:D ratio. The potential for L1EN orientational bias to propagate within CLIQUEs could conceivably result in FAPs separated by more than $10 \mathrm{~kb}$ to be 


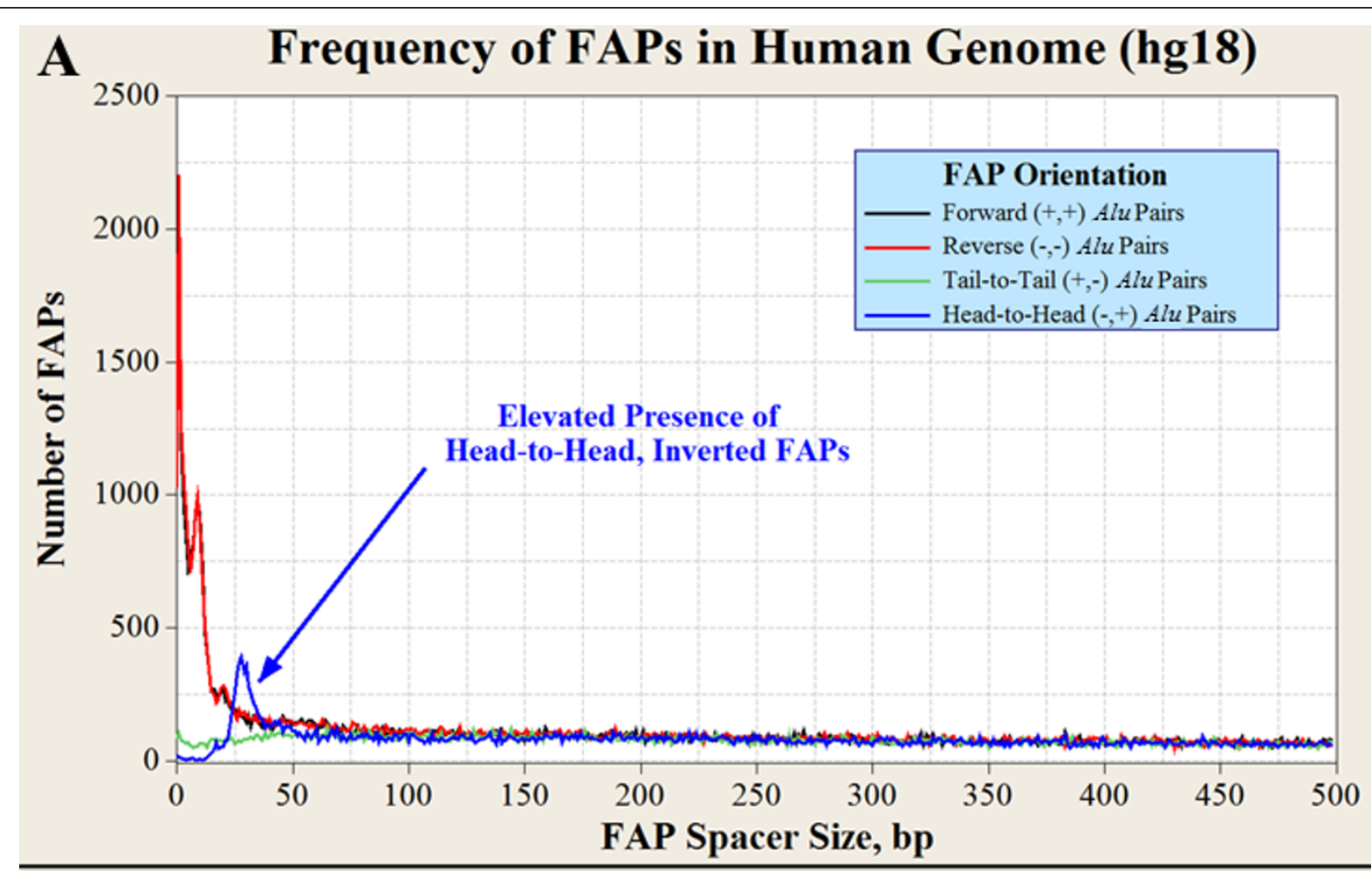

\section{B Region of Elevated Human Head-to-Head FAP Frequency}

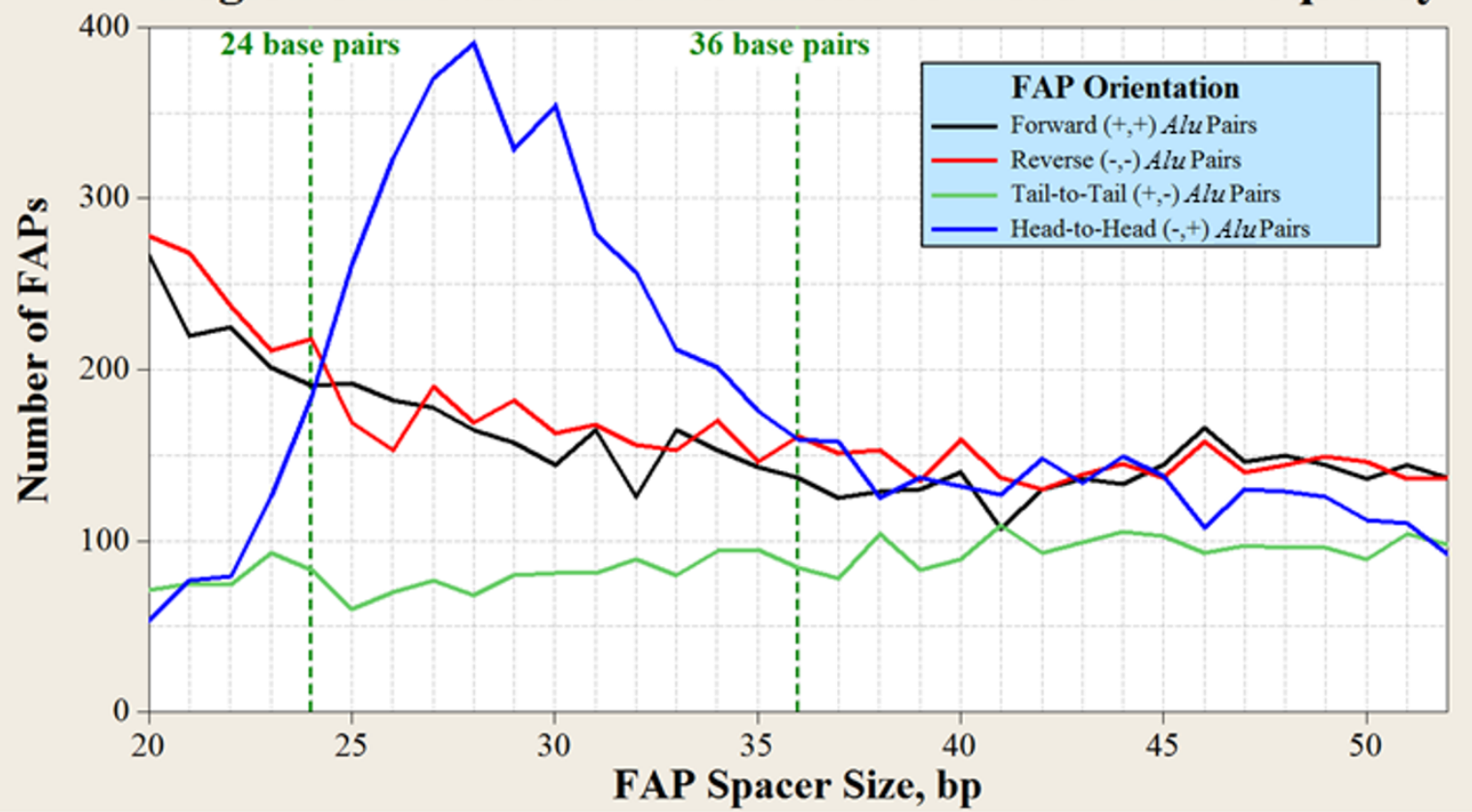

Figure 1 Frequency of closely spaced FAPs. (A) Human adjacent FAP frequency versus the spacer size (bp) separating the two members of the FAP. The number of inverted pairs (blue and green lines) is much lower than the number of direct pairs (red and black lines) when the spacer has a size $\leq 24$ bp (l:D = 0.076); (B) Spacer lengths within 24 to 36 bp define the only region within the human genome where head-to-head (inverted) FAPs outnumber either type of direct oriented FAPs. BP: base pair; FAP: full-length Alu pair. 
orientationally related. As an example, CLIQUE number 397,134 (chrX:74,530,726-74,548,236) is 17,511 bp in length and contains two full-length Alu elements which form a FAP in the forward orientation with a spacer size of 11,870 bp. This potential for orientational bias between Alu elements residing within the same CLIQUE has resulted in their exclusion for determination of genome-wide FAP I:D ratios. The adjacent FAP I:D ratio, excluding FAPs generated within the same CLIQUE, reduces the FAP sample size from 560,485 to 460,588. This correction increases the adjacent FAP I:D ratio from 0.821 to 0.955 . The smaller sample size for CLIQUE corrected adjacent FAPs slightly decreases the precision for detection of non-random I:D ratios from 0.9947 to 1.0053 to 0.9942 to $1.0058(P<0.05)$. However, the CLIQUE-adjusted adjacent I:D ratio $(0.955)$ remains statistically different from random $(P<$ $0.00001)$ even though it varies with spacer size. The most closely spaced 10 percent of human adjacent FAPs ( ppacer size $=51-205 \mathrm{bp})$ have an I:D ratio of 0.898 while the most distantly spaced 10 percent (spacer size $=$ approximately 7,400-50,000 bp) have an I:D ratio of 0.989. This relationship is illustrated in Additional File 1, Figure S6.

A calculated 52.6 percent of human LINE1, Alu and SVA sequences reside in CLIQUEs. The average CLIQUE is 1,169 bp in length and is occupied by 3.3 elements. The median CLIQUE length is $638 \mathrm{bp}$ and 95 percent of all CLIQUEs have lengths less than 4,100 bp. The most CLIQUE-rich chromosome is the chromosome 19 (0.252 CLIQUES per $\mathrm{kb})$ and the least rich is chromosome Y (0.061 CLIQUEs per kb). Over half of the longest 100 CLIQUEs are found on chromosome X, with the longest being over 55,000 bp at locus chrX:75,592,945-75,648,671 (Additional File 1, Figure $\mathrm{S} 5)$.

\section{Non-adjacent Alu pair}

One of the findings in this study is that the FAP I:D imbalance is not limited to adjacent FAPs. Intervening Alu elements within the spacer of a FAP also generate non-random FAP I:D ratios. This non-random I:D imbalance $(P<0.05)$ was detected in FAPs whose spacer contains up to 106 intervening Alu elements and over $350,000 \mathrm{bp}$. Taken at the whole human genome level, the human FAP I:D imbalance window encompasses \pm 107 of an Alu's neighboring Alu elements (Methods). No size constraint was placed upon intervening Alu elements. Therefore, while the entire inventory of human $A l u$ elements is used in this study, only I:D ratios for FAPs are reported. The smallest CLIQUE adjusted FAP sample size $(460,588)$ occurs for adjacent FAPs. Sample size ranges of 551,764 to 557,454 exist for all FAP families with more than three intervening Alu elements within the spacer (Additional File 1, Table S1). The inclusion of FAPs with intervening Alu elements requires terminology for defining different FAP types (Figure 2 and Methods).

\section{I:D ratio versus Alu pair sequence number}

Adjusting the adjacent $(0,1)$ FAP population for CLIQUEs increases its median spacer size from 930 to 1,296 bp. The CLIQUE-adjusted I:D ratios for the smaller and larger spacer sizes about this new median are 0.951 and 0.959 , respectively. Both of these I:D ratios are outside of the 0.9918 to 1.0082 range which would be expected for a random distribution $(P<0.05)$. The small difference between these I:D ratios raises the possibility that FAPs with much larger spacers may also be subject to an FAP I:D imbalance. Unfortunately, this hypothesis is difficult to measure using only adjacent FAPs as 95 percent of this population has spacer sizes of less than $11,005 \mathrm{bp}$.

The inclusion of intervening Alu elements within FAP spacers permits identification of the boundaries of the FAP I:D imbalance phenomenon (Figure 2). The FAP I: $D$ ratio as a function of $A l u$ pair sequence number (APSN) are shown in Figure 3. Both unadjusted and CLIQUE-corrected I:D curves are provided in this figure. Figure 3A shows FAP I:D ratios across APSN values of $\pm 1,000$ and reveals that the FAP I:D ratio depression appears to be limited to APSNs of $\leq 100$. Further refinement of this I:D depression boundary was accomplished by grouping 10 consecutive APSNs together. This increased the FAP sample size from approximately 555,000 to over 5.5 million. The larger sample size improved the precision of detection of the I:D depression boundary to an APSN value of \pm 107 (Methods).

Over 50 million FAPs reside within the CLIQUEadjusted FAP I:D imbalance window. Based on the CLIQUE-adjusted I:D values illustrated in Figure 3, human direct FAPs outnumber inverted FAPs by 629,027 (Additional File 1, Table S1). Random variation reduces this difference to $613,924(P<0.05)$. Figure $3 \mathrm{C}$ magnifies Figure $3 \mathrm{~A}$ to APSN values of \pm 15 and illustrates that the greatest departure between CLIQUE-adjusted and unadjusted FAP I:D ratios occurs for APSNs of less than five. The largest APSN for a FAP residing within a single human CLIQUE is 0,31 . Consequently, no CLIQUE adjustments to the FAP I:D ratio are required for APSN values greater than 31 .

\section{PCR evidence of Alu pair exclusions in the chimpanzee genome}

We have presented computational evidence for a significant FAP I:D ratio imbalance in the human genome. To investigate our hypothesis that this imbalance may be due to the increased instability of inverted $\mathrm{Alu}$ pairs, resulting in APEs, we compared the human 


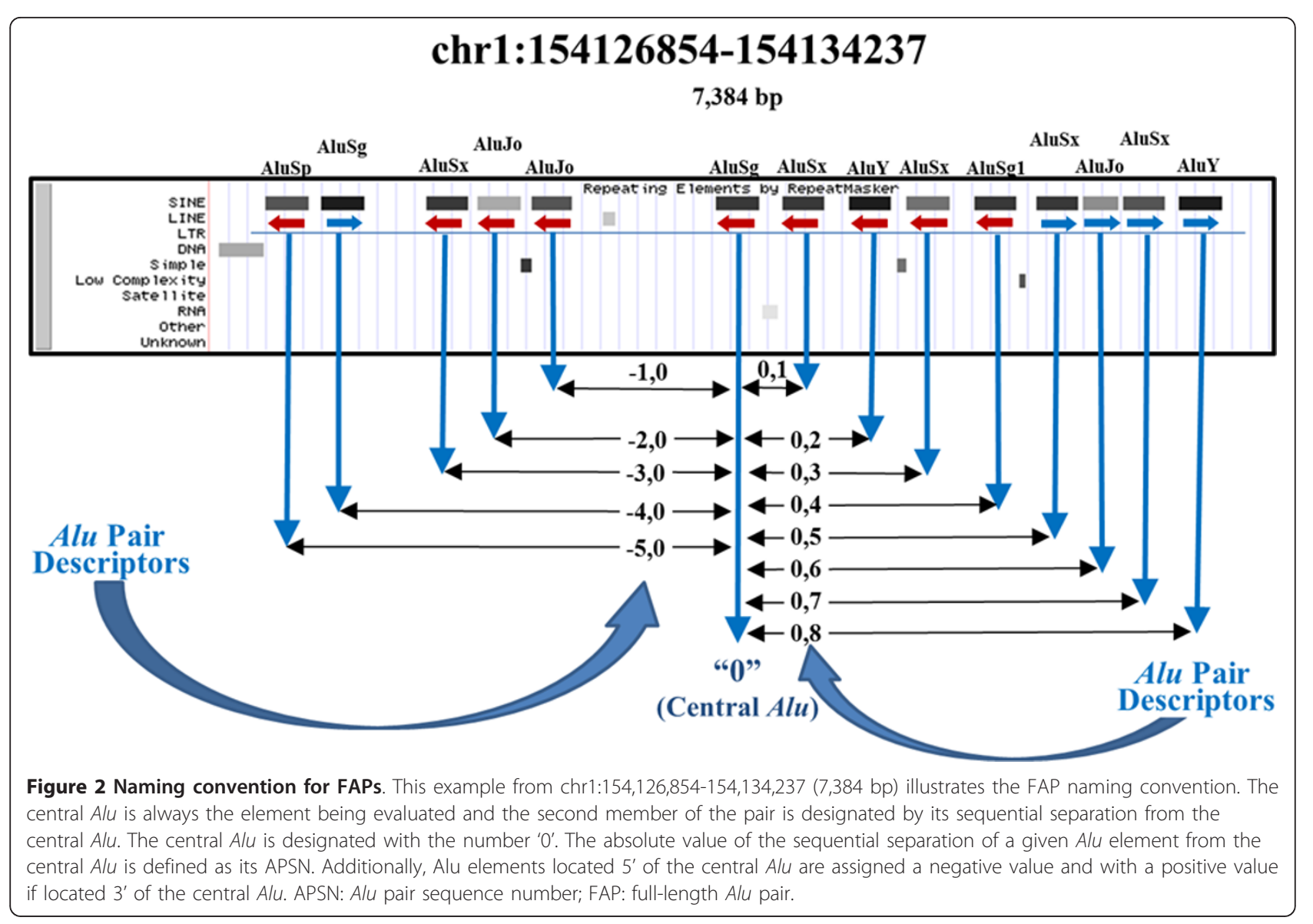

genome (hg18) to the chimpanzee genome (panTro2) to identify potential APE deletions. A total of 58 APE deletion candidate loci were identified for evaluation by PCR (Methods) in the chimpanzee genome through comparison of the human, chimpanzee, orangutan and rhesus macaque genome draft sequences. Fourteen of these loci were selected for PCR examination. These validations confirmed that 10 of these 14 loci had undergone chimpanzee-specific deletions consistent with inverted FAP instability. PCR primer design was problematic for the remaining four loci. No instances of false positive identification of chimpanzee-specific deletions were observed. The characteristics of the 10 loci confirmed as chimpanzee-specific deletions are summarized in Table 1. Images of gel chromatographs of the experimental interrogation of five of the loci are shown in Figure 4.

A secondary purpose of these PCR examinations was to assess the accuracy of the hg18 and panTro2 genome assemblies at loci involved in APE deletions. If we broadly assume that the combined hg18/panTro2 genome assemblies provide at least $50 \%$ accuracy in identification of inverted APE deletion loci, the probability of successfully validating five of these events in five consecutive PCR evaluations would be $P=0.03125\left(0.5^{5}\right)$. The fact that we were able to validate 10 such APE events in 10 consecutive PCR reactions with no evidence of false positives provides over $95 \%$ confidence that these two assemblies are at least 74 percent accurate $\left(0.74^{10}=\right.$ 0.04924). When we compared the PCR-based estimate of chimpanzee-specific inverted APE deletions to the computationally derived estimate of human inverted APE deletions for this same data set, we found these results to be within 15 percent of each other (108 versus 94). The computation was based upon the human FAP I:D ratio (0.931) for loci satisfying the original PCR criteria (Methods). Thus, these data provide strong evidence for the existence of APE-induced genomic deletions. The characteristics of the 10 loci confirmed as chimpanzee-specific deletions are summarized in Additional File 1, Table S4. Images of gel chromatographs of the experimental interrogation of five of the loci are shown in Figure 4.

Chimpanzee-specific APE deletions within these (human) orthologous loci were estimated to have occurred during the six million years following the divergence between human and chimpanzee lineages [32]. 


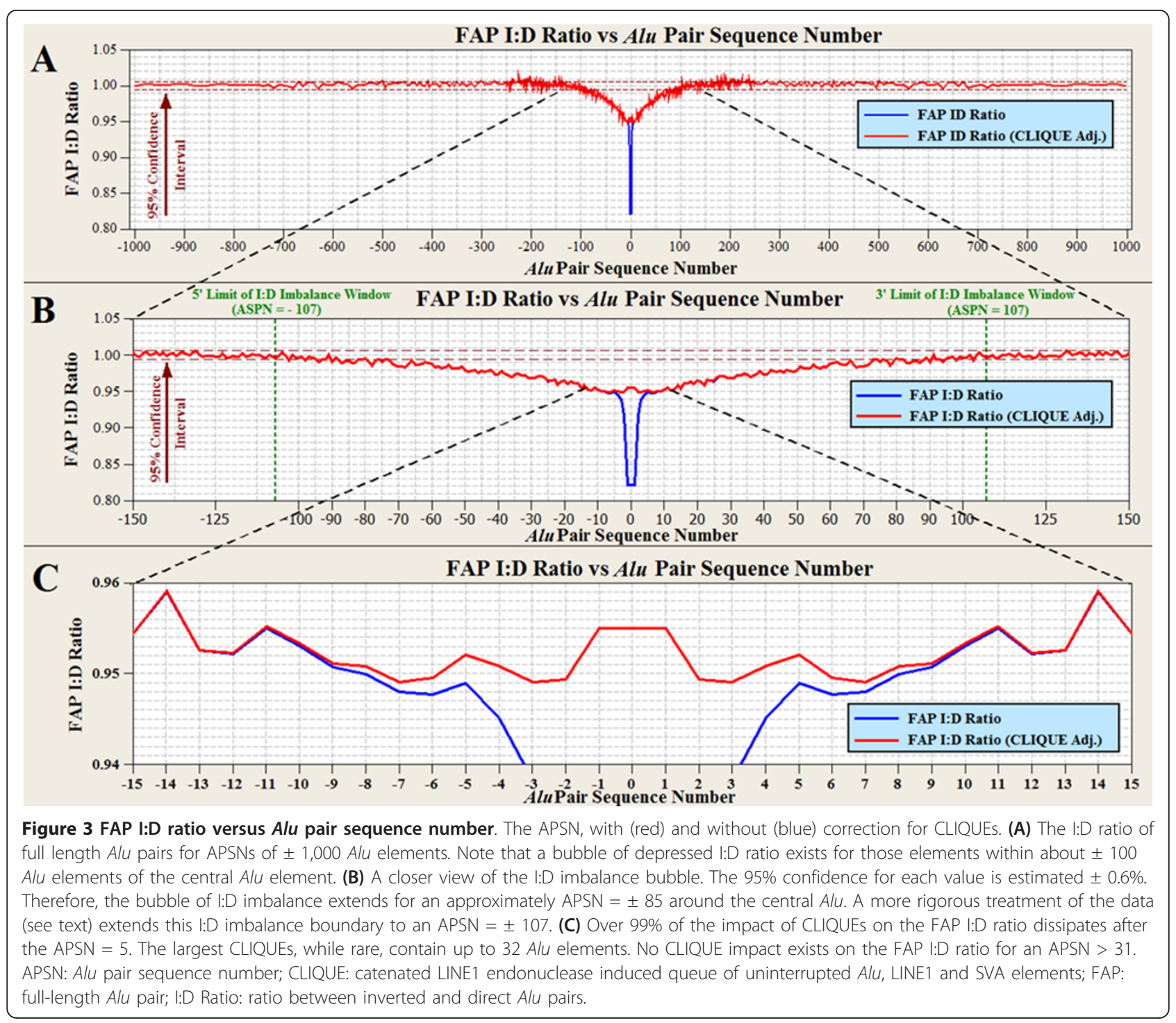

\section{Comparison of orthologous human-chimpanzee direct and inverted FAP loci}

An effort was made to better compare the characteristics of deletions within direct and inverted FAP loci. Loci selection criteria for this evaluation were identical to those used for PCR validation with two exceptions: direct FAP loci were included and chimpanzee loci were limited to those that were 1,000 to $2,000 \mathrm{bp}$ shorter than their human ortholog. The second constraint was applied to avoid lengthy deletions that could be more difficult to analyze and to provide a reasonable sample size for manual analysis. Surprisingly, these criteria generated an almost equal number of shorter direct (193) and inverted (187) chimpanzee orthologs. A subsequent examination of the shorter direct chimpanzee FAP loci revealed that inverted APE-related deletions can plausibly be attributed to 93
(48\%) of these shorter orthologous loci. These deletions are consistent with an interaction between a member of the direct FAP and a flanking Alu element in the opposite orientation. Furthermore, excluding chimpanzee orthologs that are shorter because of a human-specific retrotransposon insertion, fully 75 percent of the balance of the shorter chimpanzee loci can be plausibly attributed to have resulted from a flanking inverted APE-related deletion (see Methods and Additional File 1, Table S2). The attribution of shorter chimpanzee orthologs to possible inverted APErelated deletions is based upon the hypothesized APE deletion mechanism involving the resolution of Aluinduced double-strand breaks outlined in Additional File 1, Figure S7. This hypothesized APE deletion pattern applies to interactions between inverted FAPs with spacer sizes over $50 \mathrm{bp}$. 
Table 1 Chimpanzee-specific APEs characterized by PCR

\begin{tabular}{|c|c|c|c|c|c|c|c|c|c|}
\hline \multirow{2}{*}{$\begin{array}{l}\text { Locus } \\
\text { ID }\end{array}$} & \multirow[t]{2}{*}{ Position (hg18) } & \multicolumn{3}{|c|}{ 5' Alu Element } & \multirow{2}{*}{$\begin{array}{l}\text { Spacer } \\
\text { (bp) }\end{array}$} & \multicolumn{3}{|c|}{ 3' Alu Element } & \multirow{2}{*}{$\begin{array}{l}\text { Chimpanzee deletion Size } \\
\text { (bp) }\end{array}$} \\
\hline & & Subfamily & $\begin{array}{l}\text { Length } \\
\text { (bp) }\end{array}$ & Strand & & Subfamily & $\begin{array}{l}\text { Length } \\
\text { (bp) }\end{array}$ & Strand & \\
\hline 1 & $\begin{array}{c}\text { chr1:105842254- } \\
105848252\end{array}$ & AluY & 300 & Positive & 1,407 & AluJb & 291 & Negative & 4,896 \\
\hline 2 & $\begin{array}{c}\text { chr4:54368003- } \\
54376671\end{array}$ & AluSx & 297 & Negative & 1,292 & Alus $x$ & 310 & Positive & 5,829 \\
\hline 3 & $\begin{array}{c}\text { chr2:68246922- } \\
68253405 \\
\end{array}$ & AluY & 312 & Negative & 1,237 & AluY & 304 & Positive & 3,413 \\
\hline 4 & $\begin{array}{c}\text { chr5:71966234- } \\
71974703\end{array}$ & Alusa & 293 & Negative & 1,012 & Alusg & 310 & Positive & 4,307 \\
\hline 5 & $\begin{array}{c}\text { chr13:64130795- } \\
64137788\end{array}$ & AluJo & 297 & Positive & 1,312 & AluSx & 292 & Negative & 2,776 \\
\hline 8 & $\begin{array}{l}\text { chr17:65716901- } \\
65723822\end{array}$ & Alusg & 303 & Positive & 1,285 & AluY & 300 & Negative & 5,585 \\
\hline 9 & $\begin{array}{c}\text { chr8:53032075- } \\
53037664\end{array}$ & AluSx & 309 & Positive & 973 & AluSx & 307 & Negative & 2,340 \\
\hline 10 & $\begin{array}{c}\text { chr1:16314268- } \\
16319666\end{array}$ & Alusg & 296 & Positive & 793 & Alusq & 309 & Negative & 1,907 \\
\hline 14 & $\begin{array}{c}\text { chr5:78401563- } \\
78406842 \\
\end{array}$ & Alusa & 313 & Positive & 665 & AluSx & 301 & Negative & 1,656 \\
\hline 15 & $\begin{array}{c}\text { chr4:68494452- } \\
68500177\end{array}$ & AluY & 318 & Negative & 1,121 & AluSx & 286 & Positive & 1,654 \\
\hline
\end{tabular}

APE: Alu pair exclusion; bp: base pair; PCR: polymerase chain reaction.

\section{Discussion}

Non-random differences between direct and inverted FAPs exist for spacer sizes of zero to $\leq 350,000 \mathrm{bp}$. These differences may reflect orientation biases for either Alu element insertions or deletions. The instability of Alu pairs with spacer sizes below 650 bp has been previously described [26]. Our research suggests that additional mechanisms may be operational.

\section{APE mechanisms}

Four separate mechanisms are theorized for generating APEs within the human genome (Figure 5). Although some overlap likely exists for the spacer size ranges wherein these four mechanisms operate, the first three mechanisms appear to be confined to adjacent FAPs that are separated by $\leq 100 \mathrm{bp}$. The first of these smallspacer APEs is identified by the observation that inverted Alu pairs form near-palindromic sequences that are vulnerable to hairpin formation and can induce double-strand breaks. This mechanism is termed 'hairpin APE' (Figure 5) and is thought to be operational between spacer sizes of 0 and approximately100 bp [25].

The second mechanism is termed 'TSD APE' and appears to be active for spacer lengths of less than 23 bp (Figure 1B). This spacer length only slightly exceeds the 7 to $20 \mathrm{bp}$ size range for TSDs [2]. The nexus of high FAP density coupled with low I:D ratio is unique to human FAPs with these spacer lengths. The instability of inverted Alu pairs with spacer lengths of $\leq$ $100 \mathrm{bp}$ has been demonstrated in a yeast model [25]. This instability would be expected to reduce the FAP I: $D$ ratio. However, the coincident phenomena of high FAP density and low FAP I:D ratio may also be associated with the TPRT insertion mechanism. Alu elements inherently provide an increased density of L1EN target sites. These target sites are generated by $A l u$ TSDs and by the adenine-rich region within Alu elements [36] (see also Additional File 1, Alu-Alu Insertions). The additional L1EN target sites coupled with Alu insertion bias associated with the RNA/DNA hybrid during the TPRT mechanism are consistent with the two superimposed patterns observed in Figure 1A. The instability of inverted Alu pairs almost certainly contributes to the low I:D ratios associated with closely spaced human FAPs. However, attribution of this instability to the entirety of the low I:D ratio observed for FAPs with spacer sizes of $\leq 20$ bp may be an overestimate.

The third small-spacer APE mechanism is termed 'head-to-head APE' and involves the elevated frequency of head-to-head FAPs present between spacer sizes of 23 and $50 \mathrm{bp}$. This elevated frequency is more pronounced for spacer sizes between 25 and 35 bp and very pronounced for spacer sizes of 27 to $30 \mathrm{bp}$. Within the spacer range of 25 to $35 \mathrm{bp}$, head-to-head (inverted) FAPs outnumber either type of direct-oriented FAPs (Additional File 1, Figure S2). For spacer sizes of 27 to 

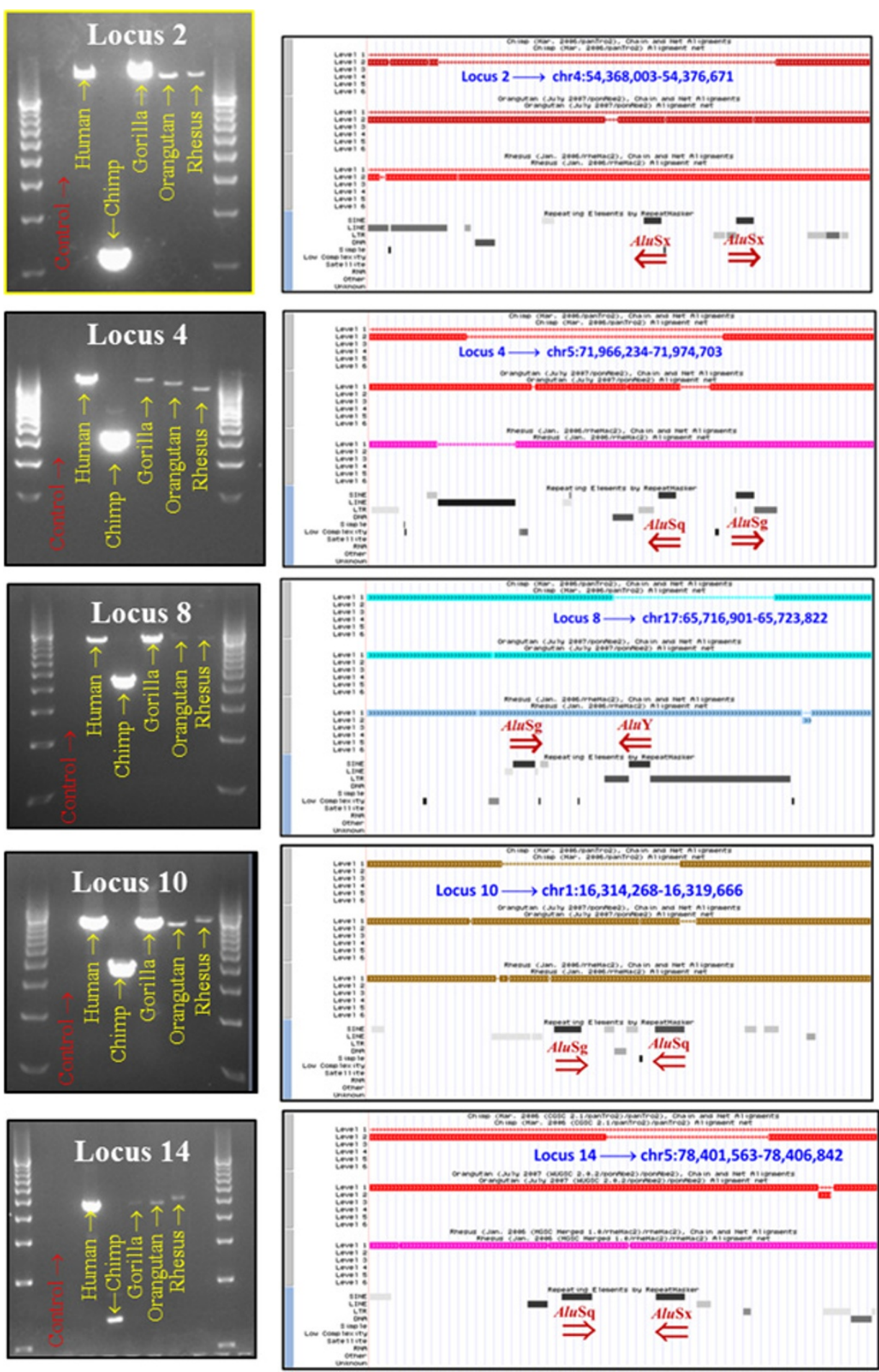

Figure 4 Chimpanzee-specific APE deletions. PCR analysis confirmed chimpanzee-specific APE deletions in orthologous human, chimpanzee, gorilla, orangutan and rhesus macaque loci. Human adjacent inverted FAP loci were chosen with spacer sizes between 651 and 1500 bp and a minimum of 1,000 bp of Alu-free flanking sequence. PCR loci were selected for which the chimpanzee loci were $>350$ bp shorter than the human ortholog. Using identical primers, PCRs were then prepared for human, chimpanzee, gorilla, orangutan and rhesus macaque. APE: Alu pair exclusion; bp: base pair; FAP: full-length Alu pair; PCR: polymerase chain reaction. 


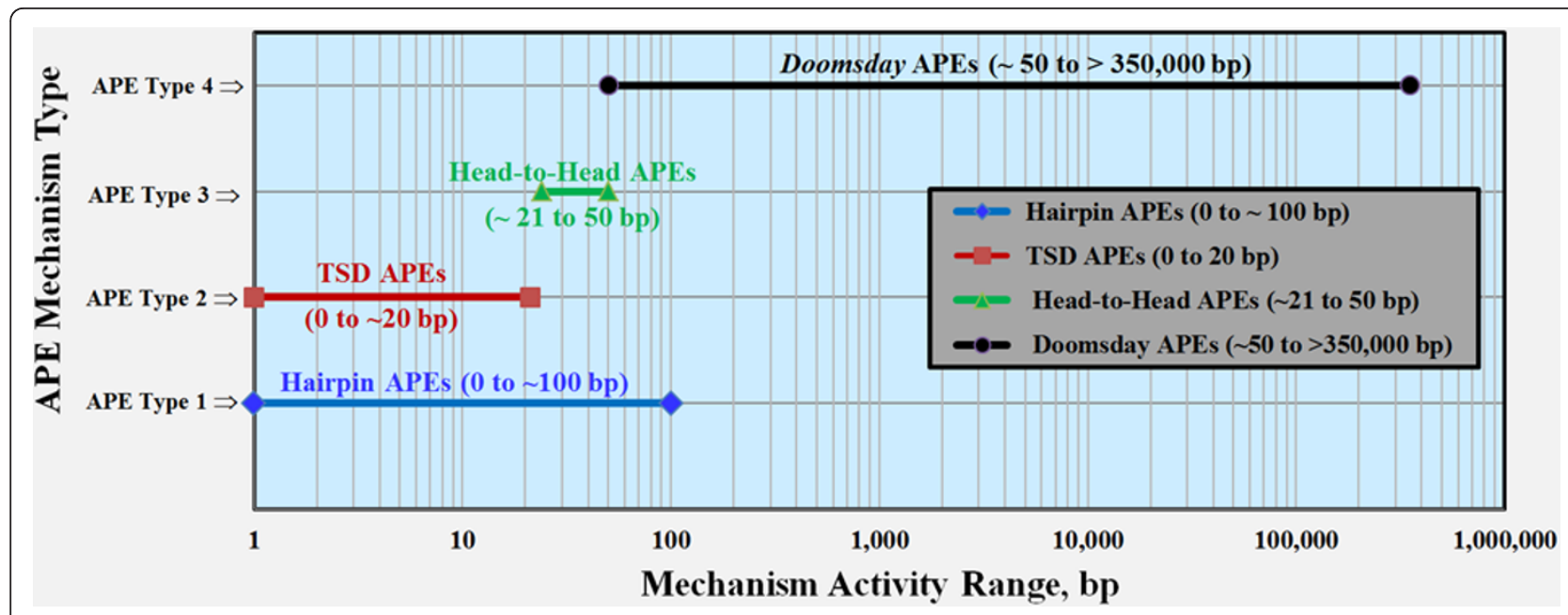

Figure 5 Estimated ranges for four potential APE mechanisms for FAPs. This semi-log chart illustrates the activity of the one previously identified [25] and three new APE mechanisms. The APE Type 1 mechanism can also be termed 'hairpin APEs' and has been previously identified as related to Alu-Alu hairpin formation with subsequent deletion. The range of this mechanism has been demonstrated to extend up to $100 \mathrm{bp}$ in a yeast model [25]. The APE Type 2 mechanism can be described as TSDs APEs' and refers to a potential orientational insertion preference for Alu element insertions within the TSD of existing Alu elements. This mechanism would preferentially form direct-oriented FAPs. As with TSD APEs (Type 2), the Type 3 APE mechanism appears to reflect an insertional preference for the formation of head-to-head (inverted) FAPs. Type 3 APEs occur approximately within the range of 21 to $50 \mathrm{bp}$ (Figure 1). The proposed mechanism for formation of Type 4 APEs is described in Figures 6 and S7 and is hypothesized to arise through a DNA conformation termed a 'doomsday junction'. APE: Alu pair exclusion; bp: base pair; FAP: full-length Alu pair.

$30 \mathrm{bp}$, head-to-head FAPs actually outnumber the sum of both direct-oriented FAP pair types. If direct-oriented FAPs are relatively stable entities, this region of elevated head-to-head frequency may evidence an insertionrelated phenomenon. A more detailed discussion of this possibility is provided in Additional File 1 (Possible Epigenetics Associated with Head-to-Head FAPs with Spacer Sizes of 24-36 bp).

The fourth APE mechanism is very dissimilar from the first three small-spacer APE mechanisms in that it involves the loss of inverted FAPs separated by approximately 50 to $\leq 350,000 \mathrm{bp}$. The third APE mechanism overlaps this range up to a spacer size of 100 bp. Over 99 percent of all CLIQUE-corrected FAPs (not residing within the same CLIQUE) have spacer sizes greater than $100 \mathrm{bp}$. The higher energy state required for formation of single-stranded DNA makes hairpin loop formation a rare event between inverted Alu pairs separated by more than $100 \mathrm{bp}$ $[25,37]$. Three possible pathways for interactions of distantly separated inverted FAPs are illustrated in Figure 6 and Additional File 1, Figure S7. Each of these pathways results in the ectopic annealing of single-stranded DNA associated with inverted FAPs. This annealing, which is hypothesized to result in a 'double-bubble' type structure, could potentially overcome the thermodynamic hurdle associated with singlestranded large-spacer hairpins. This structure is termed a 'doomsday junction' or DDJ (illustrated in Figure 6, Steps 6A and 6B and Additional File 1, Figure S7, Step 5).

Nuclease attack of DNA hairpins has been found to occur at the base, rather than the loop of DNA hairpins in yeast [23]. If DDJs exist, and if single-strand nucleases are active in primates, the eight single-stranded sections of DNA on the periphery of DDJs (Figure 6, Steps 6A and $6 \mathrm{~B}$ and Additional File 1, Figure S7, Step 5) could form attractive nuclease targets. Such nicking could help resolve the DDJ. However, this nicking could potentially result in various combinations of flanking deletions on either side of the two Alu elements forming the DDJ. The resultant tell-tale deletion patterns that we would predict from this mechanism are outlined in Additional File 1, Figure S8. The varied repair products from nuclease attack on these single-stranded structures could result in partial or total removal of one or both Alu elements. These proposed patterns are consistent with those observed by PCR of possible chimpanzee-specific APE deletions shown in Figure 4 and Additional File 1, Figure S8D. The pattern is also consistent with deletion patterns in 199 of 380 orthologous human-chimpanzee FAP loci (51\%) where a potential chimpanzee deletion had occurred (Additional File 1, Table S2). This deletion pattern increases to 75 percent when the 114 humanspecific retrotransposon insertions are removed from the data set. 


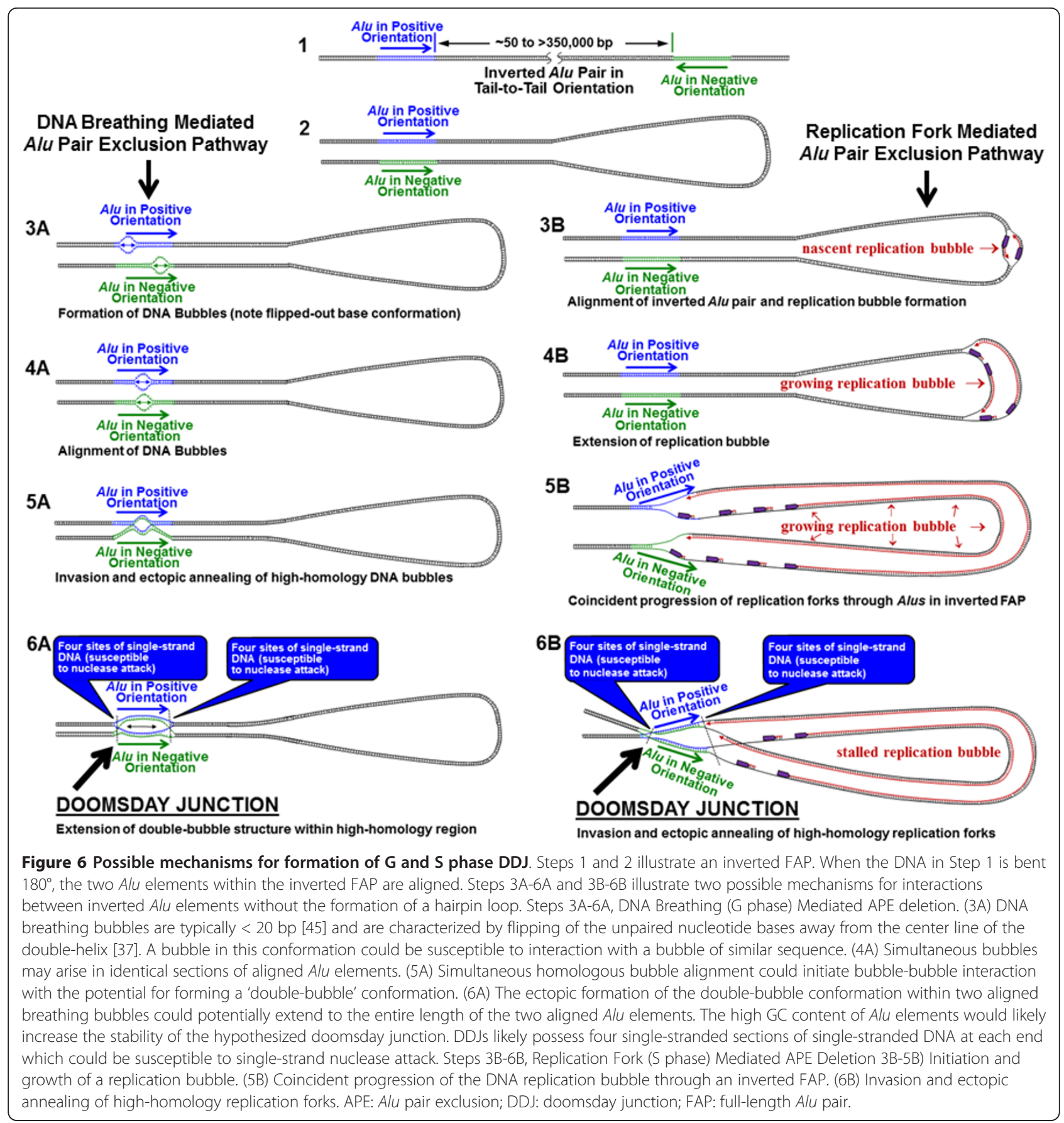

\section{G-phase doomsday APEs}

Figure 6 and Additional File 1, Figure S7 outline separate mechanisms by which DDJs could form during the $G$ and $S$ phases of the cell cycle. We propose that Gphase DDJs result from the ectopic invasion and annealing of high-homology bubbles associated with DNA breathing (Figure 6, Steps 1-6A). Nucleosomes and other chromatin structures mitigate DNA breathing and thus may reduce the potential for G-phase DDJ formation. Therefore, in addition to their multifarious roles in signaling and protein binding, nucleosomes may also serve to minimize the interaction between highhomology DNA strands. The instability of closely spaced inverted $A l u$ elements shown here and noted by previous researchers may be evidence that nucleosomes are either absent from hairpin prone DNA sequences or provide insufficient interference for hairpin formation $[3,25,26]$. The postulated G phase DDJ phenomenon 
may enjoy this same dominance over nucleosome interference.

If simultaneous DNA breathing bubbles were to arise between aligned homologous sequences, the flippedout conformation of complimentary bases on both strands could provide additional potential for intrastrand interaction (Figure 6, Step 4A) [10]. This altered genomic structure formed by the hypothetical interaction between two homologous DNA bubbles would effectively create the double-bubble conformation associated with DDJs. The initial smaller double-bubble structure (Figure 6, Step 5A) could easily expand to form a larger double-bubble which could extend to almost the entire length of the two aligned Alu elements (Figure 6, Step 6A). The high GC content (> $60 \%$ ) of Alu elements composing the large bubble conformation would likely enhance the stability of the hypothesized DDJ.

\section{S-phase doomsday APEs}

$S$ phase DDJs are proposed to result from invasion and subsequent annealing of high-homology DNA replication forks (Figure 6, Steps 1-6B and Additional File 1, Figure S4). Coincident passage of replication forks through inverted FAPs could provide an environment susceptible to formation of an S-phase DDJ. Unlike the chromatin interference present in G phase, replicating S-phase DNA is forced to lift its chromatin kimono and becomes much more vulnerable to ectopic DNA interaction. While single-strand binding proteins stabilize single-stranded portions of the replication fork, they are eventually displaced with a newly replicated strand of single-stranded DNA. This second strand could conceivably be supplied from an invading second replication fork.

Notably, upon formation of an S-phase DDJ, the DNA replication apparatus would be completely assembled and could potentially proceed, albeit in an ectopic fashion, and conceivably generate segmental duplications. In addition, the double-bubble binding of near-homologous Alu elements within a DDJ could invite the activity of cellular mismatch repair mechanisms. Such mismatch activity could help explain elevated mutation rates which have previously been observed close to deletions [38].

Finally, the DDJ mechanisms outlined in Figure 6 and Additional File 1, Figure S7 do not preclude interactions between direct-oriented FAPs. However, the distinctive ' $V$ ' shape of replication forks may provide steric hindrance to interactions with direct pairs and thus preferably favor interactions between inverted pairs. Regardless of the mechanism(s) associated with the human FAP I:D ratio imbalance, this metric is not an absolute measure of change in the number of either direct or inverted FAPs, but of the relative change between the two types.

\section{Conclusions}

Direct and inverted FAPs are distributed non-randomly in the human genome. This non-random pattern exists for APSNs $\leq 107 \mathrm{bp}$ and for spacer sizes up to 350,000 bp. A total of 59,357,435 FAPs (CLIQUE corrected) reside within this window and direct FAPs outnumber inverted FAPs by 629,027 (over two percent). Random variation only reduces this imbalance to $613,924(P<$ 0.05). Outside of CLIQUEs, no known orientation insertion preferences exist for Alu elements. We believe that APE-related deletions may be responsible for a substantial proportion of the imbalance of over 600,000 between inverted and direct human FAPs. Future investigations of the APE phenomenon should better illuminate the mechanisms involved and characterize its extent in primate genomes.

\section{Methods}

\section{Data acquisition and management}

Data used in the research was obtained from the RepeatMasker [39] output for the hg18, 2006 Human Genome assembly. This data was downloaded from the UCSC genome BLAT Table Browser http://genome. ucsc.edu/cgi-bin/hgTables[40] and imported to Excel 2010 (Microsoft Corporation; Redmond, Washington). Orthologous chimpanzee, orangutan and rhesus macaque loci were obtained using the panTro2, ponAbe2 and rheMac2 genomes assemblies, respectively. Statistics were calculated using Minitab 15 (Minitab Inc.; State College, Pennsylvania).

\section{Histogram of human Alu size distribution}

The RepeatMasker scan of the hg18 human genome assembly identifies potential $A l u$ fragments as small as $12 \mathrm{bp}$. Using a haploid genome size of $3.1 \times 109 \mathrm{bp}$, a total of 185 instances of a given $12 \mathrm{bp}$ should randomly occur in human DNA. However, most Alu elements have sequence identities between 65 and 85 percent [26]. Using the lower sequence identity (65\%) increases the number of random instances of a $12 \mathrm{bp}$ target sequence occurring in the human genome from 185 to 32,485 (Additional File 1, Figure S1). The target sequence must increase in length to 26 bp before statistical significance $(P<0.05)$ occurs. This sequence size increases to 29 bp for $60 \%$ identity. For this study, only Alu sequences of $\geq 30 \mathrm{bp}$ are used. For perspective, a 30 bp Alu fragment length is roughly 10 percent of the length of a full-length $A l u$ element. Finally, it should be noted that the $12 \mathrm{bp}$ sequences become significant $(P<$ 0.05 ) when a segment of DNA shorter than $4,770 \mathrm{bp}$ is being evaluated. 
Sequences of less than $30 \mathrm{bp}$ in length cannot be reliably determined to be actual $A l u$ elements and are therefore excluded from this truncated percentage. A lower size limit of 275 bp is set to avoid I:D ratio directional bias caused by fragmented elements that can be generated by $A l u$ insertions into a preexisting $A l u$ element (Additional File 1). The upper Alu element size limit of $325 \mathrm{bp}$ is set to avoid the potential for confounding results by inclusion of the smaller population of larger elements.

\section{Terminology for non-adjacent Alu pairs}

The central Alu in this naming convention is always designated with the number ' 0 '. The second member of the pair is designated by its sequential separation from the central Alu. If this second member of a pair is located 5' of the central Alu element, it is designated by a negative number and by a positive number if it is located 3' of the central Alu element. The value of the sequential separation of a given $A l u$ element from the central $A l u$ is defined as its APSN. For adjacent elements, these FAP pairs are described as $-1,0$ and 0,1 . Similarly, FAPs separated by 25 intervening Alu elements are described as $-26,0$ and 0,26 pairs, respectively.

\section{Determination of $95 \%$ confidence interval for FAP I:D ratios}

FAP sample sizes used in this study range from 555,354 to 567,242 (APSNs 0,1 to 0,107 ). These sample sizes are retrieved by counting functions within the Alu element Excel spreadsheet. Following removal of FAPs residing within the same CLIQUE (CLIQUE-adjusted), these data set sizes are reduced to between 460,588 and 557,364. CLIQUE-adjusted samples sizes below 550,000 only exist for APSNs $\leq 4$. For a FAP sample size of 550,000, the number of direct and inverted FAPs should range between 274,272 and $275,728(P<0.05)$. Any imbalance in direct or inverted FAPs is offset by an equal and opposite imbalance in the other FAP type. Therefore, the I:D ratio for a sample size of 550,000 is expected to range from 0.9947 to $1.0053(P \leq 0.05)$. This range increases to between 0.9942 and 1.0058 for the lowest sized $(0,1)$ FAP family of 460,588.

\section{Determination of maximum APSN within the FAP I:D ratio imbalance window}

Determination of the limits of the FAP I:D ratio imbalance boundary beyond an APSN of approximately 85 (Figure 3B) was accomplished by increasing the precision of the method. This added precision was achieved by increasing the FAP sample size. This larger sample size was acquired by calculating a 10-point moving average of the FAP I:D ratio across consecutive APSNs beyond the \pm 85 range. This approach increased the
FAP sample size from approximately 550,000 to 5.55 million and reduced the 95 percent confidence interval for randomness from $1 \pm 0.0053$ to $1 \pm 0.0017$. The highest ten consecutive APSNs which had an I:D average outside of these new confidence limits was the APSN range 103 to 112 . The midpoint of this range is the APSN value of 107 .

\section{Determination of maximum spacer size within the FAP I:D ratio imbalance window}

Approximately 90 percent of the adjacent FAPs have spacer sizes below $6,400 \mathrm{bp}$. In addition, the I:D ratio for the upper 10 percent of this family is 0.9838 which is lower than the statistically significant I:D ratio of $1 \pm$ 0.995. Consequently, determination of the boundary of the FAP I:D imbalance bubble (Figure 3B) requires examination of larger APSN families. The number of FAPs within a given size range can be summed across various APSNs. This summation was used to determine the spacer size boundaries for the FAP I:D imbalance window.

APSN families smaller than 0,25 contain very few members with spacer sizes between 300,000 and $400,000 \mathrm{bp}$. However, 3,541,238 FAPs reside within this spacer range for APSN's of 0,25 to 0,107 . This spacer size range was divided into two separate ranges of 300,000 to 350,000 and 351,000 to 400,000 . The number of FAPs within these spacer ranges was determined as 1,974,605 ( $\mathrm{I}: \mathrm{D}=0.9951)$ and 1,566,633 ( $\mathrm{I}: \mathrm{D}=0.9956)$, respectively. The expected ranges for FAP I:D ratios for these two spacer size ranges are 0.9972 to 1.0028 and 0.9969 to 1.0031 , respectively $(P<0.05)$. These two I:D ratios are outside of these ranges and thus show that the FAP I:D imbalance window extends beyond \pm $350,000 \mathrm{bp}$.

\section{Selection of loci for validation of APE deletions in the chimpanzee genome}

The methodology employed for selection of potential APE deletion loci utilized five criteria. These criteria were pair orientation, APSN, Alu element size, spacer size and $A l u$-free flanking sequence 5' and 3' of the pair being evaluated. Only inverted Alu pairs were chosen as potential experimental loci as they have been previously demonstrated to be unstable [25]. The second criterion, APSN, was limited to 0,1 (adjacent) FAPs as any intervening $A l u$ element necessarily forms a second, more closely spaced inverted pair with one of the two elements of that FAP. Therefore, any deletion associated with this locus could reasonably be attributed to interactions associated with the intervening element. For this reason, only the pool of adjacent human FAPs (APSN = 0,1 ) was used to identify candidate APE deletion loci.

The third criterion, Alu element size, was limited to the 275 to 325 bp constraints set for FAPs. The fourth 
criterion, spacer length separating the two FAP elements, was limited to those elements separated by 651 to $1,500 \mathrm{bp}$. The lower spacer size limit was set by the upper limit of previous work [26] and upper limit was set to provide an acceptable number of candidate loci. The fifth criterion, 5' and 3' Alu-free flanking sequence around a 0,1 FAP, was set to a minimum of 1,000 bp. This constraint was necessary to avoid attribution of an APE deletion to nearby elements. These criteria created locus sizes between 3,201 and 4,150 bp.

A total of 13,664 human loci were identified which satisfied these five criteria. This sample size was approximately 0.03 percent of the approximately 50 million CLIQUE-adjusted FAPs within the I:D imbalance window shown in Figure 3B. These loci were then compared to the chimpanzee panTro2 genome assembly using the LiftOver feature of the USCS Genome Browser [40-42]. This screening identified 715 (or slightly over five percent) of the chimpanzee loci that were over 350 bp smaller than their human ortholog. The less than 350 bp lower limit was set to reduce the number of false-positive loci (in other words, human specific Alu insertions can be flagged as potential sites for chimpanzee APE-related deletions). The 715 loci were individually inspected using the UCSC genome browser for the human, chimpanzee, orangutan and rhesus macaque genomes [40-44]. These inspections reduced the number of PCR candidate loci to 58. Four criteria accounted for approximately 90 percent of this reduction. These four criteria, in order of magnitude, were:

1. The presence of N's in the chimpanzee genome assembly (382 loci)

2. The insertion of a human specific transposable element as the cause of the smaller chimpanzee loci (141 loci)

3. A deletion present, but so large that it encompassed an adjacent $A l u$ element making the deletion non-diagnostic (56 loci)

4. Complementary deletions were also present in orangutan or rhesus (38 loci)

The remaining 58 loci were selected as potential candidates for further examination with PCR.

\section{Estimation of APE deletions in chimpanzee genome by observation}

Although only 58 of the 715 loci were accepted for further examination by PCR, an additional 94 of these loci showed considerable evidence of being potential APE deletions (criterions 3 and 4, above). Adding these 94 loci to the 58 PCR candidate loci increases the number of APE-related deletion loci to 152. It was also assumed that the 382 loci which contained N's in the chimpanzee (rejection criterion 1) were indeterminate and could neither be accepted nor rejected regarding detection of APE-related deletions. Separating these 382 loci (which contained N's in the chimpanzee deletion) from the original set of 715 loci reduces the total number of individually inspected loci to 333. It is estimated that 152 likely APE-related deletion loci exist out of these 333 loci (45.6\%). Of the 14 loci evaluated by PCR, 10 were informative (71.4\%). The PCR results from the remaining four loci were uninformative and no false positive instances of chimpanzee-specific deletions were observed. Combining these two probabilities provides an estimate that 32.6 percent (108) of the 333 loci were likely APE-related deletions. Therefore, within these 13,664 inverted FAP loci, a total of 108 APE-type deletions are estimated to have occurred in chimpanzee (by observation) since the human-chimpanzee divergence.

\section{Primer design for PCR}

Candidate PCR amplicon sequences were obtained with the BLAT feature of the UCSC genome browser $[40,42]$. These sequences were aligned using the BioLign software (developed by Tom Hall and available from the Buckler Lab website: http://www2.maizegenetics.net/bioinformatics). These alignments were manually inspected for common identity between the four primate species. Forward and reverse oligonucleotide primers were selected from regions of common alignment. Primer sequences are shown in Additional File 1, Table S2.

\section{PCR amplification}

All PCR amplifications were conducted in $27.5 \mu \mathrm{L}$ reactions using $25 \mathrm{ng}$ DNA template, $0.2 \mu \mathrm{M}$ oligonucleotide primer, 1.25 units TaKaRa LA Taq ${ }^{\mathrm{TM}}, 0.4 \mathrm{mM}$ dNTPs, and $1 \mathrm{X}$ TaKaRa LA Taq ${ }^{\mathrm{TM}}$ buffer containing 2.3 $\mathrm{uM} \mathrm{MgCl} 2$. A list of primers is provided in Additional File 1, Table S2. The primate panel contained templates from Homo sapiens (HeLa; cell line ATTCC CCL-2); Pan troglodytes (common chimpanzee "Clint", cell line Coriell Cell repositories NS06006), Gorilla gorilla (Western lowland gorilla; cell line Coriell Cell Repositories NG05251); Pongo abelii (Sumatran orangutan; cell line Coriell Cell Repositories NG06209); and Macaca mulatta (rhesus macaque; cell line Coriell cell Repositories NG07110). PCRs were run for $80 \mathrm{sec}$ for initial denaturation at $94^{\circ} \mathrm{C}$. Denaturing, annealing and extension times and temperatures were $20 \mathrm{sec}$ at $94^{\circ} \mathrm{C}, 20 \mathrm{sec}$ at optimum temperatures (Additional File 1, Table S2) and $8 \mathrm{~min} 30 \mathrm{sec}$ at $68^{\circ} \mathrm{C}$, respectively, for 32 cycles. The 32 cycles were followed by a final extension time of $10 \mathrm{~min}$ at $68^{\circ} \mathrm{C}$. Following amplification, all PCR products were electrophoresed on $1.5 \%$ agarose gels stained with ethidium bromide at a concentration of $1 \mu$ l per 50 $\mathrm{mL}$ of gel solution. Gels were run for 45 to $55 \mathrm{~min}$ at 
175 volts. Finally, fragments were visualized using UV fluorescence.

\section{Comparison of APE deletions in chimpanzee genome by computation and observation}

Using the original criteria for isolating potential experimental loci, 13,664 inverted FAP and 14,680 direct FAPs were identified. The I:D ratio for these FAPs is 0.931 and the difference between these inverted and direct FAPs is 1,016 , which we believe correspond to APE-associated deletion events. All Alu element insertions have occurred over the 65 million years of primate evolution. It is estimated that the most recent common ancestor of humans and chimpanzees lived approximately six million years ago [32]. Consequently, approximately 12 million years of genome evolution are estimated to have occurred between extant humans and chimpanzees. For this 12-million year period of evolution to be incorporated into calculated APE rate estimates, both orthologous chimpanzee-specific and human-specific APE-related deletions must be estimated. Only chimpanzee-specific APE-related deletions are measured in this study. Therefore, only half of the 12-million years of evolution are used (six million years) in this estimate. Therefore, a conservative estimate of 94 chimpanzee-specific APE deletions would be expected over the 6 million years since the human-chimpanzee divergence $(1016 \times 6 \div 65=94)$. This number is concordant with the 108 APE deletions previously estimated to have occurred by observational methods (see Estimation of APE Deletions in Chimpanzee Genome by Observation above).

\section{Moving average distributions of actual and random Alu clustering}

The RepeatMasker scan of the hg18 human chromosome assembly recovers 102,592 Alu elements in chromosome 1. Since orientational clustering bias has been shown to occur within CLIQUEs, only the 5' Alu element in each CLIQUE was included in this evaluation. Chromosome 1 contains 50,262 Alu elements that do not reside within a CLIQUE. Human chromosome 1 contains 34,916 CLIQUEs, of which 26,277 contain at least one Alu element. Consequently, only 76,539 $(50,262+26,277)$ Alu elements were used in this clustering evaluation. A value of +1 was assigned to each Alu on the positive strand and a value of -1 was assigned to each Alu on the negative strand. Moving average data was calculated for the 50, 100, 200, 500 and 1,000 sequential directional data points in Excel.

Five sets of 76,539 random +1 and -1 data (equivalent to the revised data set of Alu elements in human chromosome 1, above) were generated using Minitab15. This data was transferred to Excel and moving averages were calculated for each set of random data for 50, 100, 200, $500,1,000,2,000,5,000$ and 10,000 sequential directional data points. These 48 sets of moving average data (one set of actual data and five sets of random data for eight separate moving averages) were then transferred back to Minitab. Individual mean and standard deviations for each set of random distributions were determined using the Mintab15 histogram 'with fit and groups' algorithm. The five individual means and standard deviations were then averaged for each set of random moving averages. The random data curves were generated using these average mean and standard deviations (Additional File 1, Figure S3).

\section{Additional material}

Additional file 1: Supplemental Information. This file contains fundamental background information related to Alu pair exclusion research. It also contains discussions and data that support the findings within the manuscript.

Additional file 2: Definition of Terms. This file contains a list with definitions of abbreviations and novel terminology introduced within the manuscript.

\section{Abbreviations}

APE: Alu pair exclusion; APSN: Alu pair sequence number; bp: base pair; CLIQUE: catenated LINE1 endonuclease induced queue of uninterrupted Alu, LINE1 and SVA elements; DDJ: doomsday junction; dNTP:

deoxyribonucleotide triphosphate; FAP: full-length Alu pair; I:D Ratio: ratio between inverted and direct Alu pairs; LINE1: long interspersed element 1; L1EN: endonuclease domain of LINE1 ORF2 protein; L1RT: reverse transcriptase domain of LINE1 ORF2 protein; LTR: long terminal repeat; ORF: open reading frame; PCR: polymerase chain reaction; SINE: short interspersed elements; SVA: SINE/variable number of tandem repeats/Alu; TPRT: target primed reverse transcription; TSD: target site duplication; See also Additional File 2.

\section{Acknowledgements}

The authors would like to thank all members of the Batzer Laboratory for their advice and feedback. They would especially like to thank Thomas J Meyer for his insightful suggestions and sage advice in the preparation of the manuscript and figures. Special thanks are given to Jungnam Lee for her important admonition that intervening Alu elements be considered in this study of Alu pairs. This research was supported by National Institutes of Health RO1 GM59290 (MAB), and the Louisiana Board of Regents Governor's Biotechnology Initiative GBI (2002-005) (MAB).

\section{Author details}

${ }^{1}$ Department of Biological Sciences, Louisiana State University, Baton Rouge, Louisiana, 70803, USA. ${ }^{2}$ Department of Nanobiomedical Science \& WCU

Research Center, Dankook University, Chungnam 330-714, Republic of Korea.

\section{Authors' contributions}

GWC, MKK, JAW and KH designed the research; GWC and JDM performed the research; MAB contributed new reagents and analytic tools; GWC analyzed the data and wrote the paper. All authors read and approved the final manuscript.

\section{Competing interests}

The authors declare that they have no competing interests.

Received: 24 June 2011 Accepted: 23 September 2011

Published: 23 September 2011 


\section{References}

1. Lander $E S$, et al: Initial sequencing and analysis of the human genome. Nature 2001, 409(6822):860-921.

2. Cordaux R, Batzer MA: The impact of retrotransposons on human genome evolution. Nat Rev Genet 2009, 10(10):691-703.

3. Lee J, Han K, Meyer TJ, Kim HS, Batzer MA: Chromosomal inversions between human and chimpanzee lineages caused by retrotransposons. PLoS One 2008, 3(12):e4047.

4. Belancio VP, Hedges DJ, Deininger P: Mammalian non-LTR retrotransposons: for better or worse, in sickness and in health. Genome Res 2008, 18(3):343-358.

5. Luan DD, Korman MH, Jakubczak JL, Eickbush TH: Reverse transcription of R2Bm RNA is primed by a nick at the chromosomal target site: a mechanism for non-LTR retrotransposition. Cell 1993, 72(4):595-605.

6. Mathias SL, Scott AF, Kazazian HH Jr, Boeke JD, Gabriel A: Reverse transcriptase encoded by a human transposable element. Science 1991 254(5039):1808-1810.

7. Repanas K, Zingler N, Layer LE, Schumann GG, Perrakis A, Weichenrieder O: Determinants for DNA target structure selectivity of the human LINE-1 retrotransposon endonuclease. Nucleic Acids Res 2007, 35(14):4914-4926.

8. Konkel MK, Batzer MA: A mobile threat to genome stability: The impact of non-LTR retrotransposons upon the human genome. Semin Cancer Biol 2010, 20(4):211-221.

9. 1000 Genomes Project Consortium: A map of human genome variation from population-scale sequencing. Nature 2010, 467(7319):1061-1073.

10. Fogedby HC, Metzler R: Dynamics of DNA breathing: weak noise analysis, finite time singularity, and mapping onto the quantum Coulomb problem. Phys Rev E Stat Nonlin Soft Matter Phys 2007, 76(6 Pt 1):061915.

11. Batzer MA, Kilroy GE, Richard PE, Shaikh TH, Desselle TD, Hoppens CL, Deininger PL: Structure and variability of recently inserted Alu family members. Nucleic Acids Res 1990, 18(23):6793-6798.

12. Grimaldi G, Singer MF: A monkey Alu sequence is flanked by 13-base pair direct repeats by an interrupted alpha-satellite DNA sequence. Proc Natl Acad Sci USA 1982, 79(5):1497-1500.

13. Batzer MA, Deininger PL: Alu repeats and human genomic diversity. Nat Rev Genet 2002, 3(5):370-379.

14. Goodier JL, Kazazian HH Jr: Retrotransposons revisited: the restraint and rehabilitation of parasites. Cell 2008, 135(1):23-35.

15. Luan DD, Eickbush YH: RNA template requirements for target DNAprimed reverse transcription by the R2 retrotransposable element. $\mathrm{Mol}$ Cell Biol 1995, 15(7):3882-3891.

16. Kazazian HH Jr: Mobile elements and disease. Curr Opin Genet Dev 1998, 8(3):343-350

17. Morrish TA, Gilbert N, Myers JS, Vincent BJ, Stamato TD, Taccioli GE, Batzer MA, Moran JV: DNA repair mediated by endonucleaseindependent LINE-1 retrotransposition. Nat Genet 2002, 31(2):159-165.

18. Srikanta D, Sen SK, Conlin EM, Batzer MA: Internal priming: an opportunistic pathway for L1 and Alu retrotransposition in hominins. Gene 2009, 448(2):233-241.

19. Weiner AM, Deininger PL, Efstratiadis A: Nonviral retroposons: genes, pseudogenes, and transposable elements generated by the reverse flow of genetic information. Annu Rev Biochem 1986, 55:631-661.

20. Watson JB, Sutcliffe JG: Primate brain-specific cytoplasmic transcript of the Alu repeat family. Mol Cell Biol 1987, 7(9):3324-3327.

21. Quentin Y: Fusion of a free left Alu monomer and a free right Alu monomer at the origin of the Alu family in the primate genomes. Nucleic Acids Res 1992, 20(3):487-493.

22. Collins J: Instability of palindromic DNA in Escherichia coli. Cold Spring Harb Symp Quant Biol 1981, 45(Pt 1):409-416.

23. Lengsfeld BM, Rattray AJ, Bhaskara V, Ghirlando R, Paull TT: Sae2 is an endonuclease that processes hairpin DNA cooperatively with the Mre11/ Rad50/Xrs2 complex. Mol Cell 2007, 28(4):638-651.

24. Lewis $\mathrm{S}$, Akgun $\mathrm{E}$, Jasin M: Palindromic DNA and genome stability. Further studies. Ann N Y Acad Sci 1999, 870:45-57.

25. Lobachev KS, Stenger JE, Kozyreva OG, Jurka J, Gordenin DA, Resnick MA: Inverted Alu repeats unstable in yeast are excluded from the human genome. EMBO J 2000, 19(14):3822-3830.

26. Stenger JE, Lobachev KS, Gordenin D, Darden TA, Jurka J, Resnick MA: Biased distribution of inverted and direct Alus in the human genome: implications for insertion, exclusion, and genome stability. Genome Res 2001, 11(1):12-27.
27. Deininger PL, Batzer MA: Alu repeats and human disease. Mol Genet Metab 1999, 67(3):183-193.

28. Hedges DJ, Deininger PL: Inviting instability: Transposable elements, double-strand breaks, and the maintenance of genome integrity. Mutat Res 2007, 616(1-2):46-59.

29. Sen SK, Han K, Wang J, Lee J, Wang H, Callinan PA, Dyer M, Cordaux R, Liang P, Batzer MA: Human genomic deletions mediated by recombination between Alu elements. Am J Hum Genet 2006, 79(1):41-53.

30. Han $\mathrm{K}$, et al: Alu recombination-mediated structural deletions in the chimpanzee genome. PLOS Genet 2007, 3(10):1939-49.

31. Franke G, Bausch B, Hoffmann MM, Cybulla M, Wilhelm C, Kohlhase J, Scherer G, Neumann HP: Alu-Alu recombination underlies the vast majority of large VHL germline deletions: Molecular characterization and genotype-phenotype correlations in VHL patients. Hum Mutat 2009, 30(5):776-786

32. Xing J, Zhang Y, Han K, Salem AH, Sen SK, Huff CD, Zhou Q, Kirkness EF, Levy S, Batzer MA, Jorde LB: Mobile elements create structural variation: analysis of a complete human genome. Genome Res 2009, 19(9):1516-1526

33. Conrad DF, Pinto D, Redon R, Feuk L, Gokcumen O, Zhang Y, Aerts J, Andrews TD, Barnes C, Campbell P, Fitzgerald T, Hu M, Ihm CH, Kristiansson K, Macarthur DG, Macdonald JR, Onyiah I, Pang AW, Robson S, Stirrups K, Valsesia A, Walter K, Wei J, Wellcome Trust Case Control Consortium, Tyler-Smith C, Carter NP, Lee C, Scherer SW, Hurles ME: Origins and functional impact of copy number variation in the human genome. Nature 2010, 464(7289):704-712

34. Mills RE, et al: Mapping copy number variation by population-scale genome sequencing. Nature 2011, 470(7332):59-65.

35. Bailey JA, Liu G, Eichler EE: An Alu transposition model for the origin and expansion of human segmental duplications. Am J Hum Genet 2003, 73(4):823-834.

36. Levy A, Schwartz S, Ast G: Large-scale discovery of insertion hotspots and preferential integration sites of human transposed elements. Nucleic Acids Res 2010, 38(5):1515-1530

37. SantaLucia J Jr, Hicks D: The thermodynamics of DNA structural motifs. Annu Rev Biophys Biomol Struct 2004, 33:415-440.

38. Tian D, Wang Q, Zhang P, Araki H, Yang S, Kreitman M, Nagylaki T, Hudson R, Bergelson J, Chen JQ: Single-nucleotide mutation rate increases close to insertions/deletions in eukaryotes. Nature 2008 455(7209):105-108

39. Smit A, Hubley R, Green P: RepeatMasker Open-3.0 1996-2010 [http://www. repeatmasker.org].

40. Karolchik D, Hinrichs AS, Furey TS, Roskin KM, Sugnet CW, Haussler D, Kent WJ: The UCSC Table Browser data retrieval tool. Nucleic Acids Res 2004, 32 Database: D493-D496.

41. Chimpanzee Sequencing and Analysis Consortium: Initial sequence of the chimpanzee genome and comparison with the human genome. Nature 2005, 437(7055):69-87

42. Kent WJ: BLAT-the BLAST-like alignment tool. Genome Res 2002, 12(4):656-664

43. Rhesus Macaque Genome Sequencing and Analysis Consortium: Evolutionary and biomedical insights from the rhesus macaque genome. Science 2007, 316(5822):222-234.

44. Locke DP, et al: Comparative and demographic analysis of orang-utan genomes. Nature 2011, 469(7331):529-533.

45. Jeon $\mathrm{JH}$, Sung $\mathrm{W}$, Ree FH: A semiflexible chain model of local denaturation in double-stranded DNA. J Chem Phys 2006, 124(16):164905.

doi:10.1186/1759-8753-2-10

Cite this article as: Cook et al:: Alu pair exclusions in the human genome. Mobile DNA 2011 2:10 